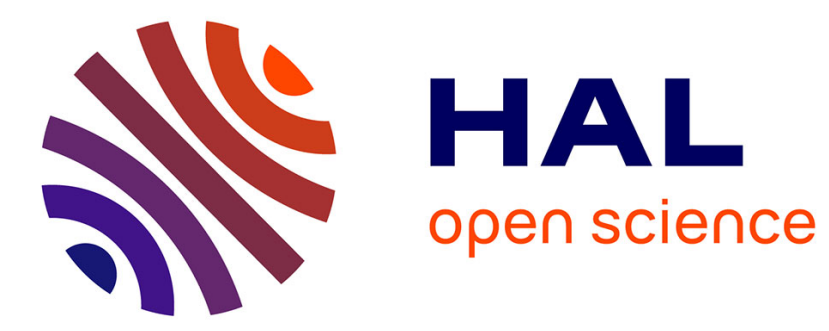

\title{
Co-evolution of pre-play signaling and cooperation
}

Francisco C. Santos, Jorge M. Pacheco, Brian Skyrms

\section{To cite this version:}

Francisco C. Santos, Jorge M. Pacheco, Brian Skyrms. Co-evolution of pre-play signaling and cooperation. Journal of Theoretical Biology, 2011, 274 (1), pp.30. 10.1016/j.jtbi.2011.01.004 . hal-00671839

\section{HAL Id: hal-00671839 \\ https://hal.science/hal-00671839}

Submitted on 19 Feb 2012

HAL is a multi-disciplinary open access archive for the deposit and dissemination of scientific research documents, whether they are published or not. The documents may come from teaching and research institutions in France or abroad, or from public or private research centers.
L'archive ouverte pluridisciplinaire HAL, est destinée au dépôt et à la diffusion de documents scientifiques de niveau recherche, publiés ou non, émanant des établissements d'enseignement et de recherche français ou étrangers, des laboratoires publics ou privés. 


\section{Author's Accepted Manuscript}

Co-evolution of pre-play signaling and cooperation

Francisco C. Santos, Jorge M. Pacheco, Brian Skyrms

PII:

S0022-5193(11)00008-7

DOI: doi:10.1016/j.jtbi.2011.01.004

Reference: YJTBI 6316

To appear in:

Journal of Theoretical Biology

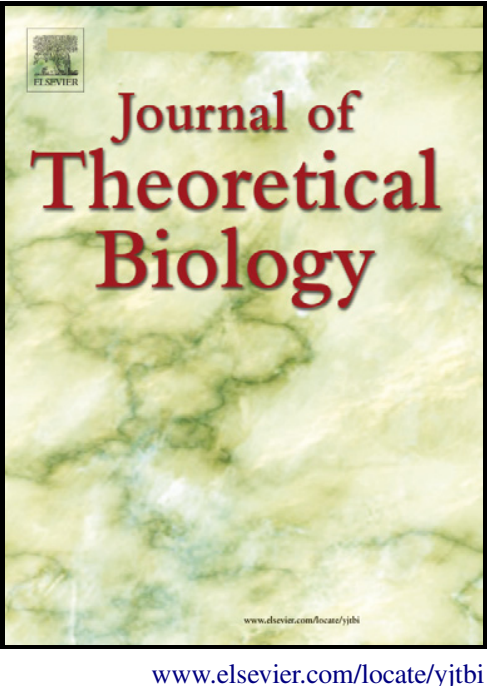

Received date: 5 October 2010

Revised date: 6 December 2010

Accepted date: 4 January 2011

Cite this article as: Francisco C. Santos, Jorge M. Pacheco and Brian Skyrms, Co-evolution of pre-play signaling and cooperation, Journal of Theoretical Biology, doi:10.1016/j.jtbi.2011.01.004

This is a PDF file of an unedited manuscript that has been accepted for publication. As a service to our customers we are providing this early version of the manuscript. The manuscript will undergo copyediting, typesetting, and review of the resulting galley proof before it is published in its final citable form. Please note that during the production process errors may be discovered which could affect the content, and all legal disclaimers that apply to the journal pertain. 


\title{
Co-evolution of pre-play signaling and cooperation
}

\author{
Francisco C. Santos ${ }^{1,2,}$, Jorge M. Pacheco ${ }^{3,2}$ and Brian Skyrms ${ }^{4}$ \\ ${ }^{1}$ CENTRIA \& Departamento de Informática, Faculdade de Ciências e Tecnologia, Universidade Nova de \\ Lisboa, 2829-516 Caparica, Portugal. \\ ${ }^{2}$ ATP-group, CMAF, Complexo Interdisciplinar, P-1649-003 Lisboa Codex, Portugal. \\ ${ }^{3}$ Departamento de Matemática e Aplicações, Universidade do Minho, 4710 - 057 Braga, Portugal. \\ ${ }^{4}$ Logic and Philosophy of Science, School of Social Sciences, University of California at Irvine, Irvine, CA \\ 92612, U.S.A.
}

$9 \quad{ }^{*}$ Corresponding author:

Francisco C. Santos

CENTRIA, Faculdade de Ciências e Tecnologia, Universidade Nova de Lisboa

Quinta da Torre 2829-516 Caparica

Portugal

Email: fcsantos@fct.unl.pt ; Tel. (+351) 212948536 FAX (+351) 212948541 
17 A finite-population dynamic evolutionary model is presented, which shows that increasing the individual capacity of sending pre-play signals (without any pre-defined meaning), opens a route for cooperation. The population dynamics leads individuals to discriminate between different signals and react accordingly to the signals received. The proportion of time that the population spends in different states can be calculated analytically. We show that increasing the number of different signals benefits cooperative strategies, illustrating how cooperators may take profit from a diverse signaling portfolio to forecast future behaviors and avoid being cheated by defectors.

Keywords: evolution; cooperation; coordination; collective action; signaling. 


\section{Introduction}

In their work on the major transitions of evolution, Maynard Smith and Szathmáry (Maynard-Smith and Szathmáry, 1995) show that in each transition, there are obstacles to the emergence of cooperation that need to be overcome. Throughout most of the book these problems are modeled as the Prisoner's Dilemma (PD) games, but near the end they remark that in some cases the Stag Hunt (SH) Game (the rowboat) (Skyrms, 2004) may be a better model. Without precise characterizations of the payoffs involved, it may sometimes be difficult to tell which is the appropriate model. Cooperative hunting provides natural examples of SH games (Boesch, 1994; Creel and Creel, 1995; Stander, 1992), but let the opportunities for free riding become ample and they may degenerate into PD games. Many popular mechanisms put forward to explain the evolution of cooperation in the PD, such as kin selection, may transform the PD into a SH (Skyrms, 2004). We treat both games together here.

Costless pre-play communication with signals that have no preexisting meaning (also known as cheap-talk) might not, on the face of it, be expected to do much. But Robson (Robson, 1990) pointed out that such signaling can destabilize the non-cooperative equilibrium in the PD. Suppose a mutant arises who can utilize an unused signal as a "secret handshake". The mutant sends the signal, cooperates with others who send it, and defects against the natives who do not send it. All goes well for the invaders until another mutant arises who sends the signal and then defects. Robson's paper was followed by others using "secret handshake" arguments to establish stability properties of the efficient Nash equilibrium in games with multiple equilibria (Kim and Sobel, 1995; Matsui, 1991; Waerneryd, 1991). In the SH game, analysis of large population (replicator) dynamics shows that pre-play signaling can not only change the stability properties of equilibria, but also can create new equilibria, and change the relative size of basins of attraction of cooperative and non-cooperative equilibria (Skyrms, 
2002). Basins of attraction were there investigated by computer simulation. Here we are able to give analytical results. We present a finite population model of evolution with mutation, in which the proportion of time spent at equilibria can be explicitly calculated. Signaling has a strong effect. In the $\mathbf{S H}$ without communication it has been long known that evolution favors the risk dominant equilibrium (Kandori et al., 1993). This is no longer true with signaling. Where the cooperative equilibrium differs from the risk dominant one, signaling can favor cooperation. Furthermore the tilt towards cooperation (in a sense we will make precise) increases with the number of signals that are present in the population. Remarkably, this remains true even for the PD.

\section{The Model}

Following (Skyrms, 2002; Skyrms, 2004), if there are $\sigma$ possible signals, one can define a strategy $A$ as a vector of the form $A=<$ signal, reaction to signal $1, \ldots$, reaction to signal $\sigma>$, creating an overall set of $n_{S}=\sigma 2^{\sigma}$ different strategies. A simple game of cooperation without signaling can hence be viewed as a game with a single common signal, $\sigma=1$. If people can only behave as cooperators $(\boldsymbol{C s})$ or defectors $(\boldsymbol{D} \mathrm{s})$, when two strategies are paired the outcome of the interaction can still be described in terms of a symmetric two-player game of cooperation, with the usual payoff matrix

$$
\begin{aligned}
& \text { C D } \\
& \begin{array}{l}
C \\
D
\end{array}\left(\begin{array}{ll}
R & S \\
T & P
\end{array}\right)
\end{aligned}
$$

In the following we shall study the role of signaling whenever $\boldsymbol{R}>\boldsymbol{T}>\boldsymbol{P}>\boldsymbol{S}(\mathbf{S H})$ and $\boldsymbol{T}>\boldsymbol{R}>\boldsymbol{P}>\boldsymbol{S}$ (PD).

Let us consider a finite well-mixed population of $Z$ interacting individuals and assume that individuals revise their behaviors by social learning, implemented by means of a stochastic 
update rule (Nowak, 2006; Sigmund, 2010; Traulsen et al., 2006; Traulsen et al., 2007). At each time step an individual $i$ with fitness $\Pi_{i}$ (characterized here by the game payoff) will update her/his strategy by imitating a randomly chosen individual $j$ with fitness $\Pi_{j}$ with a probability $p$ that increases with the increase in payoff difference between $j$ and $i$ (Traulsen et al., 2006; Traulsen et al., 2007). Hence, successful individuals will be imitated and the associated strategy will spread in the population. This probability may be conveniently written in terms of the so-called Fermi distribution (from statistical physics) $p=\left[1+e^{-\beta\left[\Pi_{j}(k)-\Pi_{i}(k)\right]}\right]^{-1}$, in which $\beta$ (an inverse temperature in physics) translates here into noise associated with errors in decision making (Traulsen et al., 2006). For high values of $\beta$ we obtain pure imitation dynamics commonly used in cultural evolution studies, whereas for $\beta \rightarrow 0$, selection becomes so weak that evolution proceeds as random drift. It is noteworthy, however, that the following results remain robust to the adoption of other update processes, such as the Moran process, in its birth-death or death-birth variant (Nowak, 2006).

We further assume that with a probability $\mu$ individuals switch to a randomly chosen strategy, freely exploring the space of possible behaviors. The ensuing analysis is largely simplified if one takes the limit of $\mu \rightarrow 0$ (so-called small-mutation limit) (Fudenberg and Imhof, 2005; Imhof et al., 2005). In the absence of mutations, the end states of evolution are inevitably monomorphic, as a result of the stochastic nature of the evolutionary dynamics and update rule(s). By introducing a small probability of mutation, the population will either end up wiping out the mutant or witness the fixation of the intruder. Hence, in the small-mutation limit, the mutant will fixate or will become extinct long before the occurrence of another mutation and, for this reason, the population will spend all of its time with a maximum of two strategies present simultaneously. 
Whenever two specific strategies are present in the population, say $\boldsymbol{A}$ and $\boldsymbol{B}$, the payoff of an individual with a strategy $\boldsymbol{A}$ in a population with $k \boldsymbol{A}$ s and $Z-k \boldsymbol{B}$ s can be written as $\Pi_{A}(k)=\frac{k}{Z} P_{A, A}+\frac{Z-k}{Z} P_{A, B}$, where $P_{A, A}\left(P_{A, B}\right)$ stands for the payoff obtained as a result of the mutual behavior ( $\boldsymbol{C}$ or $\boldsymbol{D}$ ) of an $\boldsymbol{A}$ strategist in a single interaction with a $\boldsymbol{A}(\boldsymbol{B})$ strategist. This allows one to describe the evolutionary dynamics of our population in terms of a reduced Markov Chain of size $n_{S}$ (Fudenberg and Imhof, 2005; Imhof et al., 2005), where each state represents a possible monomorphic end-state of the population associated with a given strategy, and the transitions between states are defined by the fixation probabilities of a single mutant of one strategy in a population of individuals who adopt another strategy. The resulting stationary distribution characterizes the average time the population spends in each of these monomorphic states, and can be computed analytically (see below). In the above expression of the payoffs, one is including self-interactions, which introduce an error, which may be sizeable only in very small populations. In fact, we checked that all results below hold whether or not self-interactions are included. Inasmuch as $Z>25$ the absence of selfinteractions introduces correction below $1 \%$ in the stationary distributions.

Given the above assumptions, it is easy to write down the probability to change the number $k$ of individuals with a strategy $\boldsymbol{A}$ (by \pm one in each time step) in a population of $Z-k \boldsymbol{B}$ strategists: $T^{ \pm}(k)=\frac{Z-k}{Z} \frac{k}{Z}\left[1+e^{-\beta\left[\Pi_{A}(k) m \Pi_{B}(k)\right]}\right]^{-1}$. This can be used to compute the fixation probability of a mutant with a strategy $\boldsymbol{A}$ in a population of $Z-1 \boldsymbol{B}$ s. Following (Ewens, 2004; Karlin and Taylor, 1975; Nowak et al., 2004; Traulsen et al., 2006), it is given by $\rho_{B, A}=\left(\sum_{i=0}^{Z-1} \prod_{j=1}^{i} \lambda_{j}\right)^{-1}$, where $\lambda_{j}=T^{-}(j) / T^{+}(j)$. In the limit of neutral selection $(\beta \rightarrow 0), \lambda_{i}$ becomes independent of the fitness values : $\rho_{B A}=1 / Z$. Considering a set $\left\{1, \ldots, n_{S}\right\}$ of 
different strategies, the fixation probabilities define $n_{S}^{2}$ transition probabilities of the reduced Markov Chain, with the associated transition matrix

$$
M=\left(\begin{array}{cccc}
1-\eta\left(\rho_{1,2}+\ldots+\rho_{1, n_{S}}\right) & \eta \rho_{1,2} & \ldots & \eta \rho_{1, n_{S}} \\
\eta \rho_{2,1} & 1-\eta\left(\rho_{2,1}+\rho_{2,3}+\ldots+\rho_{1, n_{S}}\right) & \ldots & \eta \rho_{2, n_{S}} \\
\ldots & \ldots & \ldots & \ldots \\
\eta \rho_{n_{s}, 1} & \ldots & \ldots & 1-\eta\left(\rho_{n_{s}, 1}+\rho_{n_{s}, 1}+\ldots+\rho_{n_{s}, n_{S}-1}\right)
\end{array}\right)
$$

with $\eta=\left(n_{s}-1\right)^{-1}$ providing the appropriate normalization factor. The normalized eigenvector associated with the eigenvalue 1 of the transposed of $M$ provides the stationary distribution described before (Fudenberg and Imhof, 2005; Imhof et al., 2005). It is also noteworthy that, as the population spends most of the time in the vicinity of monomorphic states, the fraction of time the population spends in states in which individuals cooperate with its own strategy also corresponds to the fraction of time the population spends in cooperative scenarios. Consequently the stationary distribution obtained from the matrix $M$ provides both the relative evolutionary advantage of each strategy, and also the stationary fraction of cooperative acts.

\section{Results}

\subsection{Chatting to coordinate: the stag-hunt game}

Let us consider a population of individuals interacting through a SH game (Skyrms, 2001; Skyrms, 2004) with the following payoff matrix

$$
\begin{gathered}
C \\
C \\
D \\
D \\
\left(\begin{array}{cc}
1 & -0.5 \\
0.5 & 0
\end{array}\right)
\end{gathered}
$$

Here Cooperation $(\boldsymbol{C})$ is associated with the coordinated action required to attain the highest payoff, whereas Defection (D) (or non-cooperation) counters such collective achievement, 
such that mutual defection leads to coordination into the least beneficial payoff. In the absence of different signals (that is, $\sigma=1$ ), the evolutionary dynamics associated with this game can be described by the stationary distribution obtained via the Markov Chain with 2 states $(\boldsymbol{C}$ and $\boldsymbol{D} \mathrm{s})$ and a transition matrix defined via the fixation probabilities of a single mutant. Since $\rho_{C, D}=\rho_{D, C}<1 / Z$, both $C$ s and $D$ s are protected against invasion and replacement by any other strategy. Moreover, for this particular payoff matrix $(T=0.5, R=1$, $P=0$ and $S=-T$ ), the population can be found in a cooperative or defective state with equal probabilities (see below). Hence, both cooperation and defection can be considered as Evolutionarily Stable in finite populations (so-called $\boldsymbol{E S S}_{\boldsymbol{N}}$ ), as defined in (Nowak, 2006; Nowak et al., 2004), such that the fixation probability of any of the other strategies is always smaller than neutral fixation, that is, $1 / N$.

Let us now introduce a signaling stage before each interaction $(\sigma=2)$. Individuals can now send one of two signals ("0" or " 1 ") without any pre-defined meaning, from which we obtain a Markov chain with 8 states. As all signals are considered equivalent and perfectly symmetric, all results below remain invariant if a fixed cost is assigned to each signal, apart from a trivial shift in all elements of the payoff matrix.

The results are shown in Figure 1. While the abundance of each signal in the population remains symmetric (Figure 1a), strategies that discriminate between signals prevail (Figure 1b). The most successful are those that react cooperatively to their own signal, promoting a positive feedback of their acts. As noted in (Robson, 1990), a mutant can be successful by adopting a signal that is not used in the population, and use it as a pre-play signal to ensure a posteriori coordination. This becomes clear if one analyzes the main transition probabilities between states shown in Figure 2. The arrows denote transition probabilities that are larger 
than $\rho_{n}=\frac{1}{Z}$, i.e., those that are favored by evolution, in the absence (a) and presence (b) of different signals.

While transitions between strategies of the same signal are not favored by evolution, the availability of different signals leads to new transitions between different signalers most of them favored by evolution. As shown in Figure $2 b$, these new transitions turn the two strategies, which are self-reinforcing and discriminative - i.e. strategies that react cooperatively to their own signal and defect when facing a different signal - into the only two $\boldsymbol{E S S}_{\boldsymbol{N}}$ 's, as no transition from such monomorphic states is favored by evolution. In addition, as shown in Figures $1 \mathrm{c}$ and $2 \mathrm{~b}$, cooperative strategies (blue circles) are favored by the transitions among different signals and prevail in the population.

Without different signals, the stationary fraction of cooperative acts is dictated by the size of the basins of attraction of cooperation and defection (risk dominance). With signaling, this is no longer true. Whenever different signals are available, even if costless and meaningless, full cooperation will occur $80 \%$ of the time (for the game parameters described above), contrary to $50 \%$ in the absence of different signals. Consequently individuals are able to assign a meaning to the signals and, as a result, modify the original basins of attraction. Conveying a signal "I" may be understood as, "I will go for Stag, if you signal 1" or, equivalently, the same meaning conveyed by a signal " 0 ". In practice, however, even if in most of the stable monomorphic configurations the information portrayed by each signal is clear, the meaning of each signal emerges accidently from the stochastic nature of evolution. Meanings are therefore transient and frequency dependent, co-evolving with the strategies present in the population and used at profit to ensure coordination (Skyrms, 2010). As a result, cooperation will thrive by means of the "secret handshake" (Robson, 1990) needed for coordination, without the necessity of keeping it "secret". In fact, deceiving benefits neither the sender nor 
the receiver in the SH. As a result, true signalers will perform better than any mutant and emerge as $\boldsymbol{E S S}_{\boldsymbol{N}}$ (Nowak et al., 2004), benefiting from information content enclosed in each signal.

The reasoning above leads naturally to a different but pertinent question: If transitions between distinct signals are favored by evolution and, as a result, cooperation finds a window of opportunity to thrive, a higher number of signals should enhance even more the chances of cooperators. Since the transition from defective to cooperative states demands the adoption of signals that are not present in the native population, increasing the number of available signals can only ease such process. Figure 3 confirms such hypothesis. As the number of available signals increases, transitions between distinct signals become more frequent (see Figure $3 b$ ). This will reinforce the flow of probabilities into cooperative strategies.

As shown if Figure 4 - where we portray the stationary fraction of cooperative acts as a function of the temptation to defect $T$ (with $R=1, P=0$ and $S=-T$ ) - the positive effect of signaling occurs independently of the particular payoffs of the SH game matrix. In the absence of different signals, the stationary fraction of cooperative acts is defined by the ratio $\rho_{C, D} / \rho_{D, C}$ where $\rho_{C, D}\left(\rho_{D, C}\right)$ is the fixation probability of a $\boldsymbol{C}(\boldsymbol{D})$ in a population of $Z-1 \boldsymbol{D} \mathrm{s}$ $\left(\boldsymbol{C}\right.$ ). For $0<T<0.5, \rho_{C}>\rho_{D}$ whereas the opposite occurs for $0.5<T<1.0$. It is noteworthy, that the value of $T=0.5$ also indicates the threshold above which Defection becomes the riskdominant behavior under the $\mathbf{S H}$, i.e, the best option whenever the opponent is equally likely to play $\boldsymbol{C}$ or $\boldsymbol{D}$ (Nowak, 2006). Yet, whenever the number of signals increases, this riskdominance balance is disrupted in favor of cooperation. It is as if cooperation becomes the risk dominant strategy for the entire range of parameters of the SH game. Finally, the transition at $T=0.5$ - associated with the change of the risk-dominant strategy of the $\mathbf{S H}$ becomes sharper with the increase of the intensity of selection $\beta$, likewise to the enhancing effect of cooperation through signaling (increasing function of $\beta$ ). 


\subsection{When deceiving becomes a profitable option: the Prisoner's dilemma game}

Let us now consider one population of individuals interacting through a PD with the following payoff matrix

$$
\begin{aligned}
& \text { C D } \\
& { }_{D}^{C}\left(\begin{array}{cc}
1 & -0.5 \\
1.5 & 0
\end{array}\right)
\end{aligned}
$$

Contrary to the SH dilemma, in the PD cooperators are always disadvantageous irrespectively of the fraction of cooperators. Hence, in the absence of different signals, it is not surprising

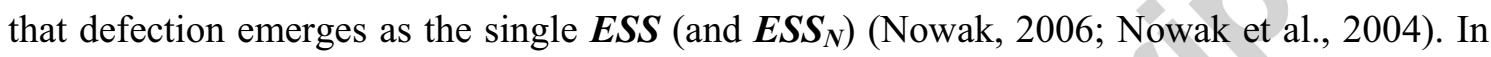
the presence of different signals, as mutual cooperation is no longer the best possible outcome in polymorphic populations, deceiving becomes an option. Hence, signals may have the same effect as in the Stag-hunt game but, contrary to it, defectors who fake signals may end up advantageous with respect to cooperators and true signalers. Life gets harder for cooperators as now they can be betrayed at profit to the traitors.

In Figure 5 we depict the transition probabilities for the case with one and two available signals, following the same convention used in the case of the SH game. For $\sigma=2$ (Figure 5b), we no longer have strategies that are $\boldsymbol{E S S}_{\boldsymbol{N}}$, as in the case of $\sigma=1$ (Figure 5a). Defection no longer works as a sink for all transition probabilities. Similarly to the $\mathbf{S H}$ game, the transitions most favored by evolution are those between states with different signals. However, now transitions between strategies with the same signal can be favored by evolution.

Let us consider two mini-games: One (A) in which transitions take place only between strategies with the same signal (solid arrows in Figure 5b) and another (B) in which one considers transitions only between different signals (dashed arrows lines in Figure 5b). Analysis of these mini-games shows that defective strategies (red circles) are the only possible $\boldsymbol{E S S}_{N}(\mathbf{I D D}$ and $\boldsymbol{O D D})$ in A whereas cooperative strategies (blue circles) are the only 
candidates for $\boldsymbol{E S S}_{N}(\boldsymbol{O C D}$ and $\mathbf{1 D C})$ in B. Thus, the overall level of cooperation results from the strength and number of transitions between different signalers, from which one can infer that increasing the number of signals will favor the prevalence of cooperative strategies. This is confirmed in Figure 6 where we show that cooperation is promoted whenever one increases the number of signals, over a wide range of parameters of the PD. Cooperation can emerge as a result of the arms race between $i$ ) the exploration of new signals by cooperators (to avoid being cheated by defectors) and $i$ ) the search of cooperative signals by defectors (to deceive cooperators). By increasing the number of signals, cooperators have a larger portfolio of signals to pick from, something they learn to use to their own advantage.

\section{Conclusion}

We have shown analytically how pre-play signaling leads to profound changes in the evolutionary dynamics of cooperative games, favoring cooperation. As kin-discrimination, beard chromodynamics or phenotypic diversity (Antal et al., 2009; Gardner and West; Jansen and van Baalen, 2006; Sigmund, 2009; Traulsen and Nowak, 2007), pre-play signaling represents an important correlation device, under which cooperation may prevail. Yet, here cooperation freely emerges from the co-evolution of signals and actions which are not built-in in the individual, addressing in a general framework the study of central aspects of Human evolution, from the self-organized drive towards an individual adoption of a given signaling system to the emergence of the latter (Skyrms, 2002; Skyrms, 2010). We analyze two important metaphors of cooperation: The Stag-Hunt (SH) (or coordination) game and the Prisoner's dilemma (PD). In coordination dilemmas, individuals willing to cooperate learn how to use the information encoded in each signal to identify other cooperators, reducing the risk of facing defection upon a cooperative act. In addition, the existence of a large number of 
signals enhances the tendency to cooperate, as it enlarges the portfolio of available signals that cooperators may use at profit to coordinate. Since mutual cooperation is always the best possible outcome, cooperators who are able to discriminate between their own strategy and the one of others are robust against the invasion of mutants. Consequently, the emergence of $\boldsymbol{E S S}_{\boldsymbol{N}}$ requires that these strategies are i) cooperative, ii) discriminative and iii) selfreinforcing, that is, they cooperate with individuals who adopt the same signal.

Remarkably, the enhancement of cooperation through signaling also applies to games where deception constitutes a profitable option, and where defection is the only stable strategy, as in the PD. In the presence of pre-play signaling, those strategies that opt invariably to defect are no longer stable in the PD. However, the same remains true for any type of cooperative strategy. Thus, in the absence of any stable strategy, the fate of cooperation emerges from the conflict between deception by fake signaling and development of reliable "secret handshakes" (Robson, 1990). These features are strongly dependent on the number of signals available, and illustrate the advantages of a complex signaling system.

\section{Acknowledgments}

The authors acknowledge the financial support of FCT-Portugal (FCS and JMP) and the Air Force Office of Scientific Research FA9550-08-1-0389 (BS). 


\section{References}

Antal, T., Ohtsuki, H., Wakeley, J., Taylor, P., and Nowak, M., 2009. Evolution of cooperation by phenotypic similarity. Proceedings of the National Academy of Sciences 106, 8597.

Boesch, C., 1994. Cooperative Hunting in Wild Chimpanzees Animal Behav. 48, 653-667.

Creel, S., and Creel, N.M., 1995. Communal Hunting and Pack Size in African Wild Dogs, Lycaon-Pictus. Animal Behaviour 50, 1325-1339.

Ewens, W.J., 2004. Mathematical Population Genetics I. Springer.

Fudenberg, D., and Imhof, L., 2005. Imitation Processes with Small Mutations. Journal of EconomicTheory 131, 251-262.

Gardner, A., and West, S., 2010. Greenbeards. Evolution 64, 25-38.

Imhof, L.A., Fundenberg, D., and Nowak, M.A., 2005. Evolutionary cycles of cooperation and defection. Proc Natl Acad Sci U S A 102, 10797-10800.

Jansen, V., and van Baalen, M., 2006. Altruism through beard chromodynamics. Nature 440, 663-666.

Kandori, M., G. , Mailath, and Rob, 1993. Learning, Mutation and Long Run Equilibria in Games. Econometrica 61, 29-56.

Karlin, S., and Taylor, H.M.A., 1975. A first course in Stochastic Processes. Academic, London.

Kim, Y.-G., and Sobel, J., 1995. An Evolutionary Approach to Pre-play Communication. Econometrica 63, 1181-1193.

Matsui, A., 1991. Cheap-talk and Cooperation in Society. Journal of Economic Theory 54, 245-258.

Maynard-Smith, J., and Szathmáry, E., 1995. The Major Transitions in Evolution. Freeman, Oxford.

Nowak, M.A., 2006. Evolutionary Dynamics. Belknap/Harvard. 
Nowak, M.A., Sasaki, A., Taylor, C., and Fudenberg, D., 2004. Emergence of cooperation and evolutionary stability in finite populations. Nature 428, 646-50.

Robson, A., 1990. Efficiency in Evolutionary Games: Darwin, Nash, and the Secret Handshake. J Theo Bio 144, 379-396.

Sigmund, K., 2009. Sympathy and similarity: The evolutionary dynamics of cooperation. Proceedings of the National Academy of Sciences 106, 8405.

Sigmund, K., 2010. The Calculus of Selfishness. Princeton University Press.

Skyrms, B., 2001. The Stag Hunt Proceedings and Addresses of the American Philosophical Association 75, 31-41.

Skyrms, B., 2002. Signals, Evolution, and the Explanatory Power of Transient Information. Philosophy of Science 69, 407-428.

Skyrms, B., 2004. The Stag Hunt and the Evolution of Social Structure. Cambridge University Press.

Skyrms, B., 2010. Signals: Evolution, Learning \& Information. Oxford University Press.

Stander, P.E., 1992. Cooperative Hunting in Lions - the Role of the Individual. Behavioral Ecology and Sociobiology 29, 445-454.

Traulsen, A., and Nowak, M., 2007. Chromodynamics of cooperation in finite populations. PLoS One 2, 270.

Traulsen, A., Nowak, M.A., and Pacheco, J.M., 2006. Stochastic dynamics of invasion and fixation. Phys Rev E 74, 011909.

Traulsen, A., Pacheco, J.M., and Nowak, M.A., 2007. Pairwise comparison and selection temperature in evolutionary game dynamics. J Theor Biol 246, 522-9.

Waerneryd, K., 1991. Evolutionary Stability in Unanimity Games with Cheap-talk. Economic Letters 36, 375-378. 


\section{Figure captions}

\section{Figure 1. Stationary distribution for the Stag-Hunt dilemma with 2 signals ("0" or "1").}

a) As expected, the evolutionary dynamics is symmetric with respect to each of the signals. b) In the limit of rare mutations, the population spends most of the time in strategies that discriminate between signals and cooperate with their own signal (patterned bars). c) the accumulated frequencies of the four strategies in which individuals cooperate with each other under a monomorphic scenario, shows that, in the presence of two signals, cooperation prevails. $(\mathrm{N}=150, \beta=0.05, \mathrm{~T}=0.5, \mathrm{R}=1, \mathrm{P}=0, \mathrm{~S}=-0.5)$.

\section{Figure 2. Transition probabilities favored by evolution a) in the absence of signaling and} b) with 2 signals available. With pre-play signaling, strategies that $i$ ) discriminate between signals and ii) react cooperatively to their own signal, become the only evolutionary stable strategies in finite populations. Arrows $\boldsymbol{A} \rightarrow \boldsymbol{B}$ denote that a mutant $\boldsymbol{B}$ in a population of $\boldsymbol{A s}$ will fixate with a probability larger than $1 / Z$. Blue (red) circles stand for strategies that opt for cooperation (defection) in a monomorphic scenario. The percentage values stand for the prevalence of each strategy associated with the stationary distribution (see also Fig. 1b). The equivalence the role played by the 2 signals of panel b), results in a transition graph with a high level of symmetry. The parameters chosen are the same as in Fig. 1.

Figure 3. Dependence in the number of different signals. a) Fraction of time the population spends in cooperative strategies as a function of the number of signals available. b) Ratio between the accumulated transition probabilities of monomorphic states with the same signal and monomorphic states with different signals, as a function of the available number of signals. The relative change with the number of signals shows how transitions 
between distinct signals become more frequent. The parameters are the same as in Fig. 1 and 2.

Figure 4. Average fraction of time spent in monomorphic cooperative states as a function the Temptation to defect $\boldsymbol{T}$ for different number of signals. When the number of signals increases, the original (risk-dominance) balance between cooperators and defectors in the absence of different signals (black line with open circles and vertical dashed line) is modified by a game setting in which cooperation becomes dominant for the entire range of the $\mathbf{S H}$ game $(\mathrm{N}=150, \beta=0.1, \mathrm{~S}=-\mathrm{T}, \mathrm{R}=1, \mathrm{P}=0)$.

Figure 5. a) Stationary probability distribution for the PD dilemma with a) $\sigma=1$ and b) $\boldsymbol{\sigma}=\mathbf{2}$. As before, arrows denote transitions favored by evolution and blue (red) circles denote strategies that lead to full cooperation under a monomorphic scenario. In the absence of different signals (a), defection is the only stable strategy. Yet, whenever two different signals are available (b), those strategies that opt invariably to defect are no longer stable. The overall level of cooperation results from the interplay between the mini-game between different signalers (pictured in solid arrows and where both cooperative strategies OCD and 1DC are stable) and the mini-game played between strategies of the same signal (dashed arrows, where the four strategies that lead to defection are stable). Parameters: $\mathrm{T}=1.5, \mathrm{~S}=-0.5, \mathrm{R}=1, \mathrm{P}=0$, $\mathrm{N}=150, \beta=0.05$.

Figure 6. Average prevalence of cooperative strategies as a function of the temptation to defect $T$ in the PD for different number of signals. Cooperation is enhanced in the entire PD regime whenever one increases the number of signals available. These results were obtained with same parameters as in Fig. 5, and with $\mathrm{R}=1, \mathrm{P}=0$ and $\mathrm{S}=1-\mathrm{T}$. 

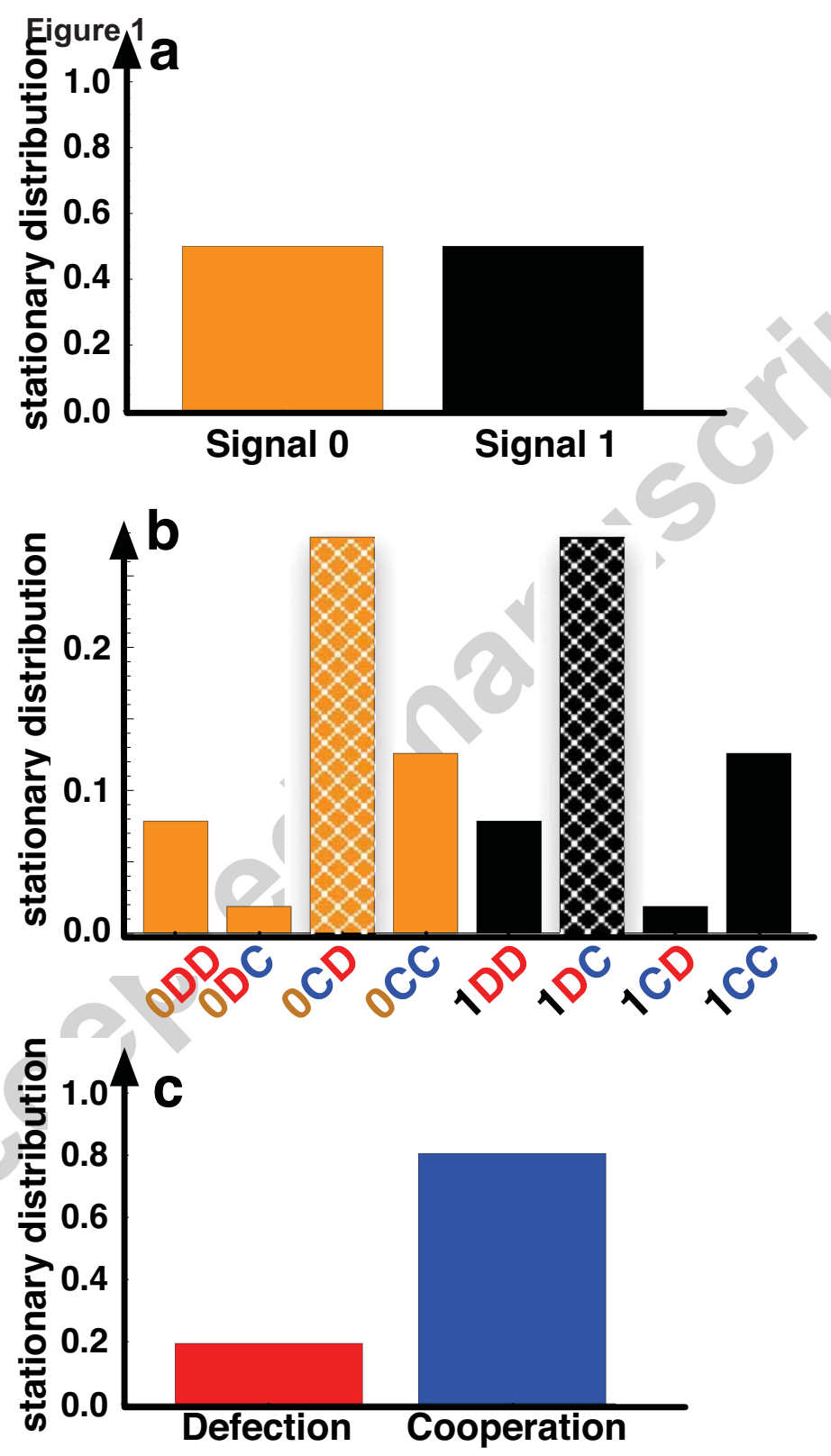

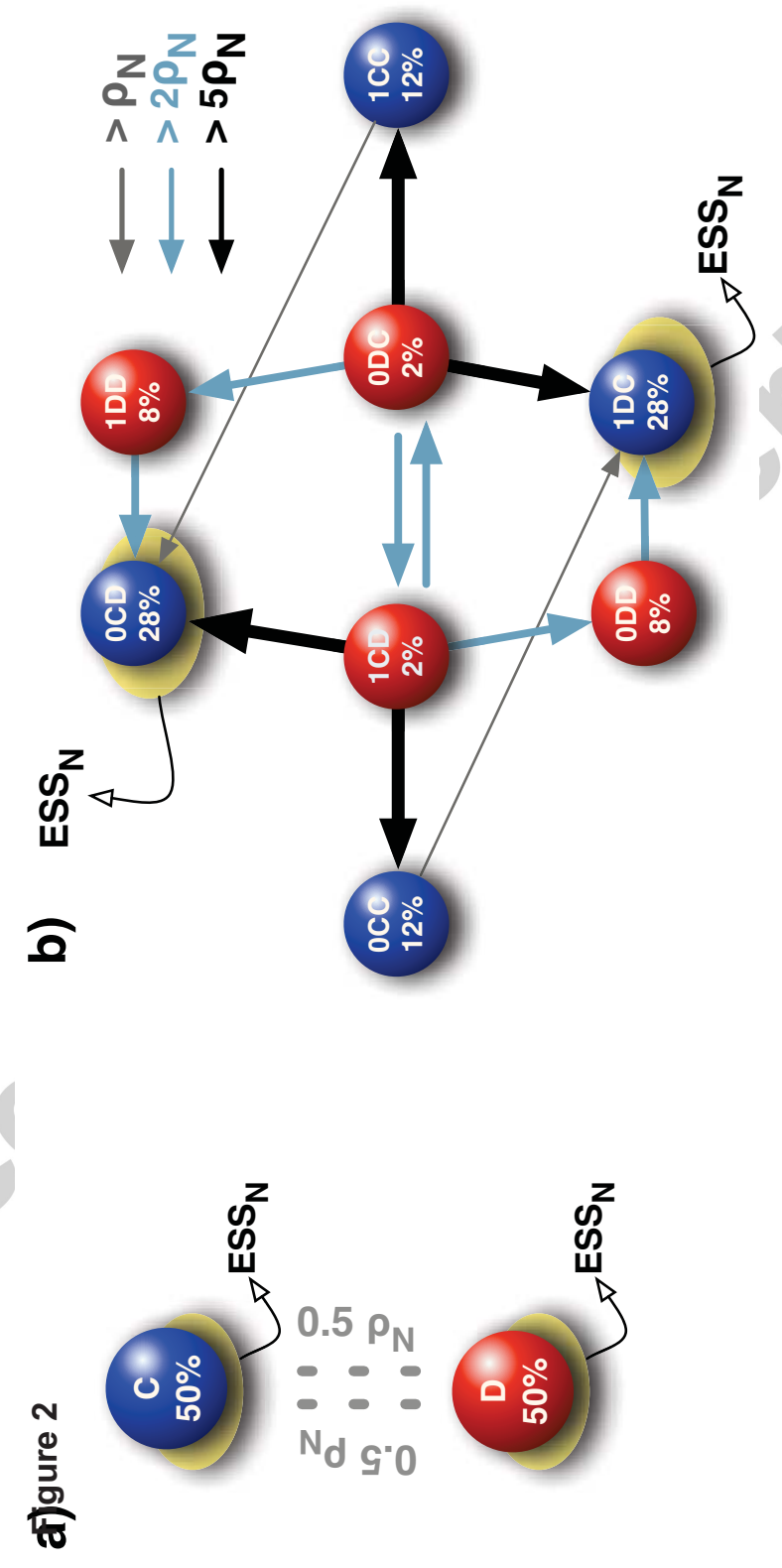


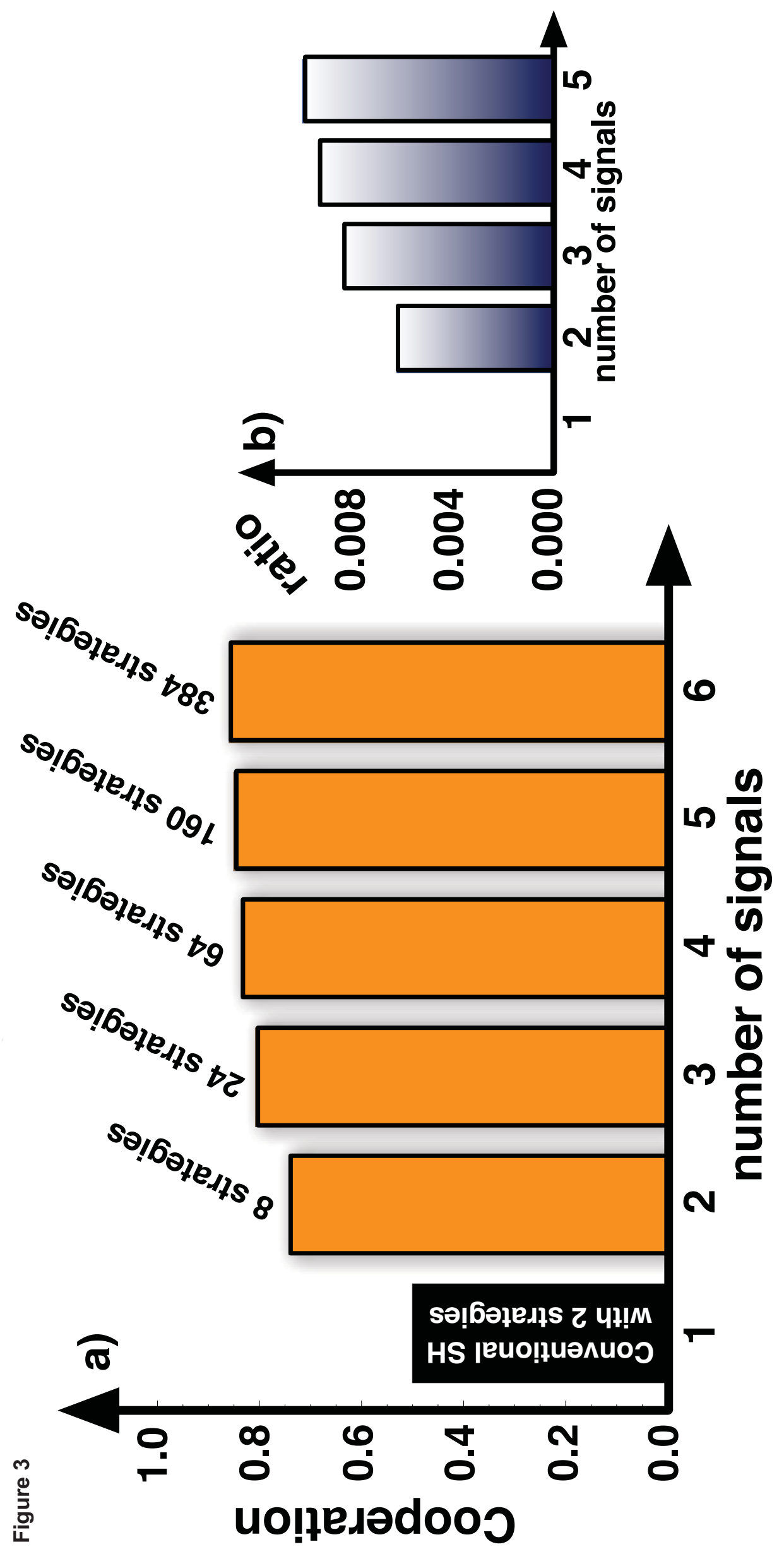




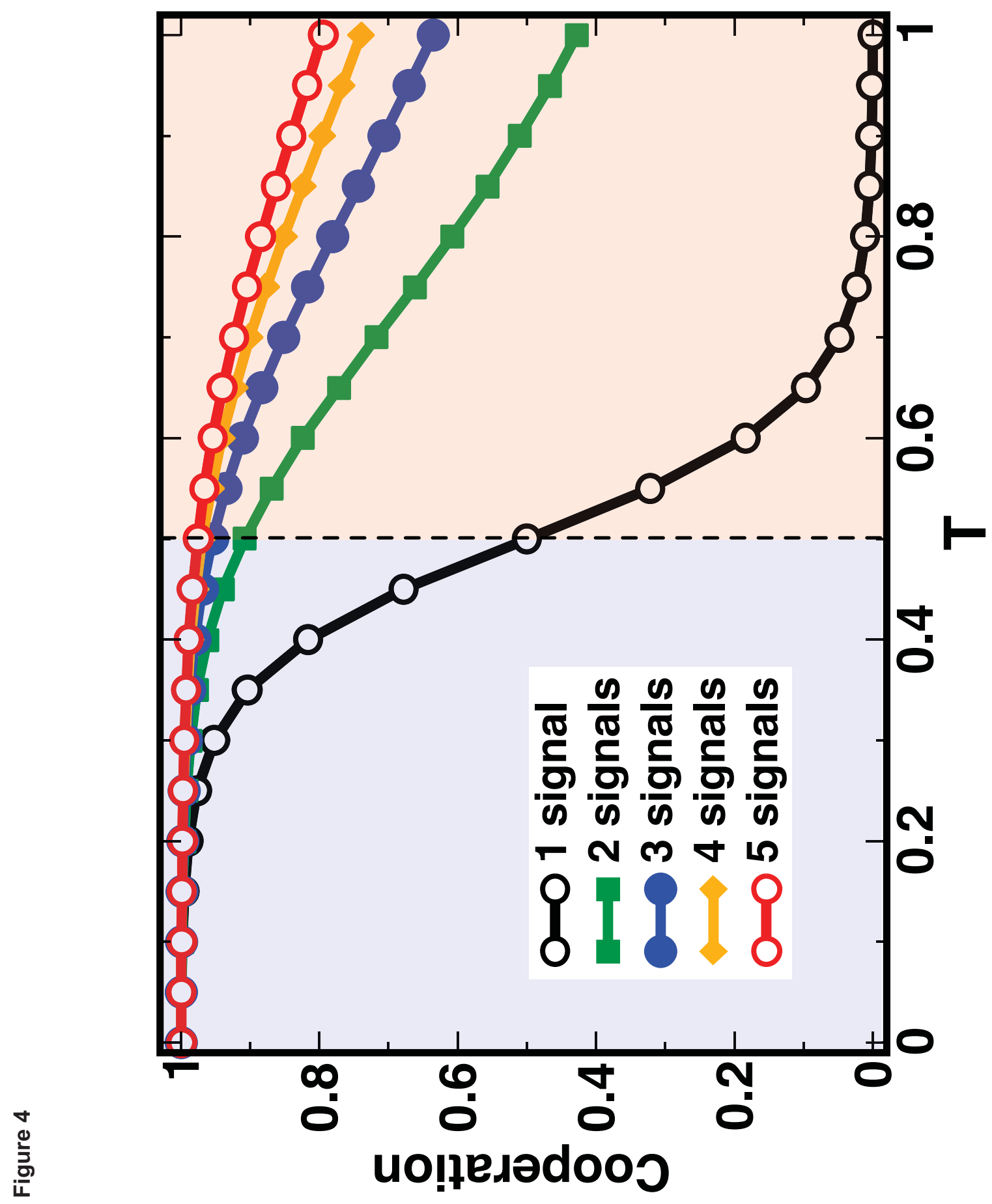




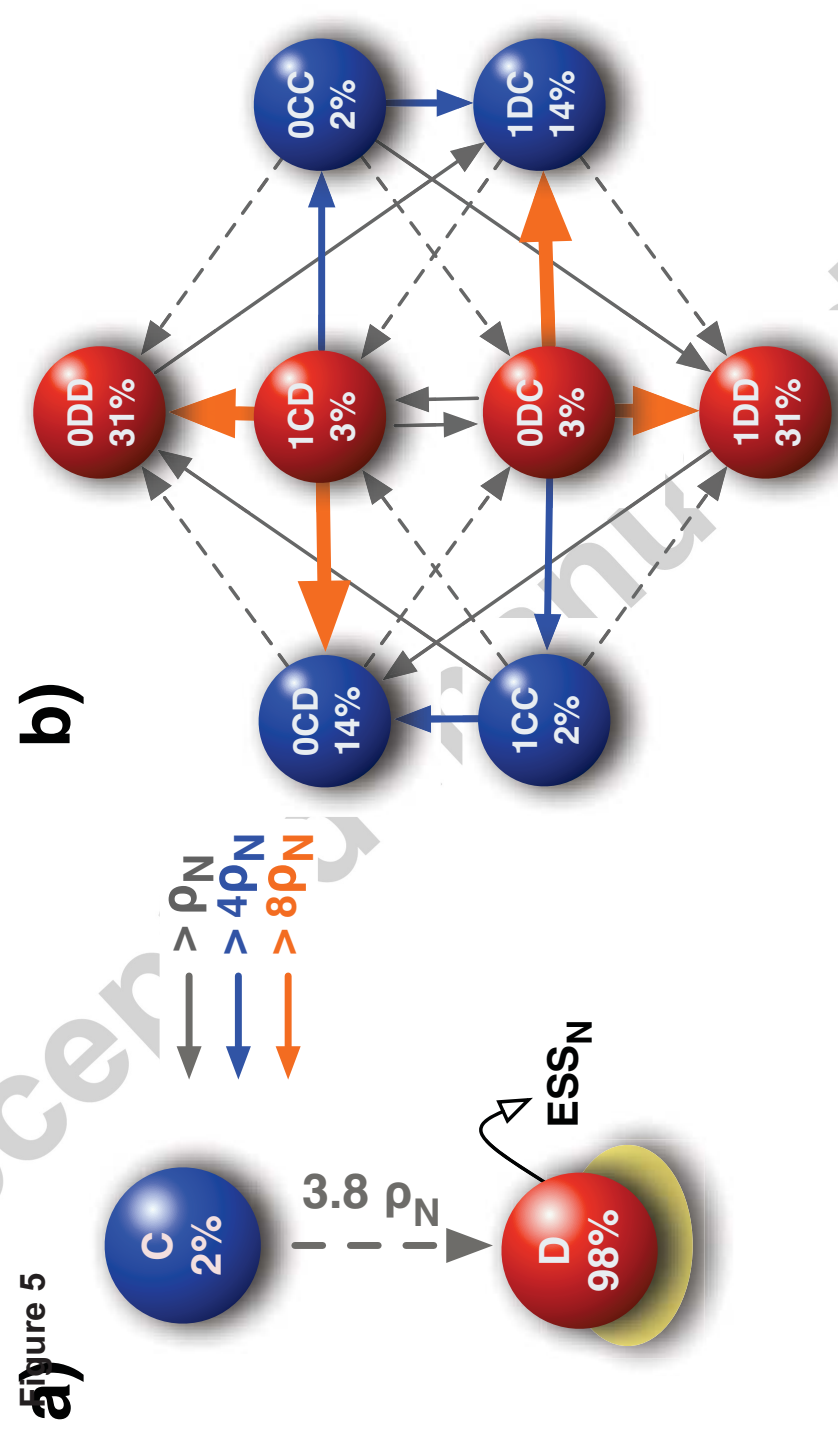




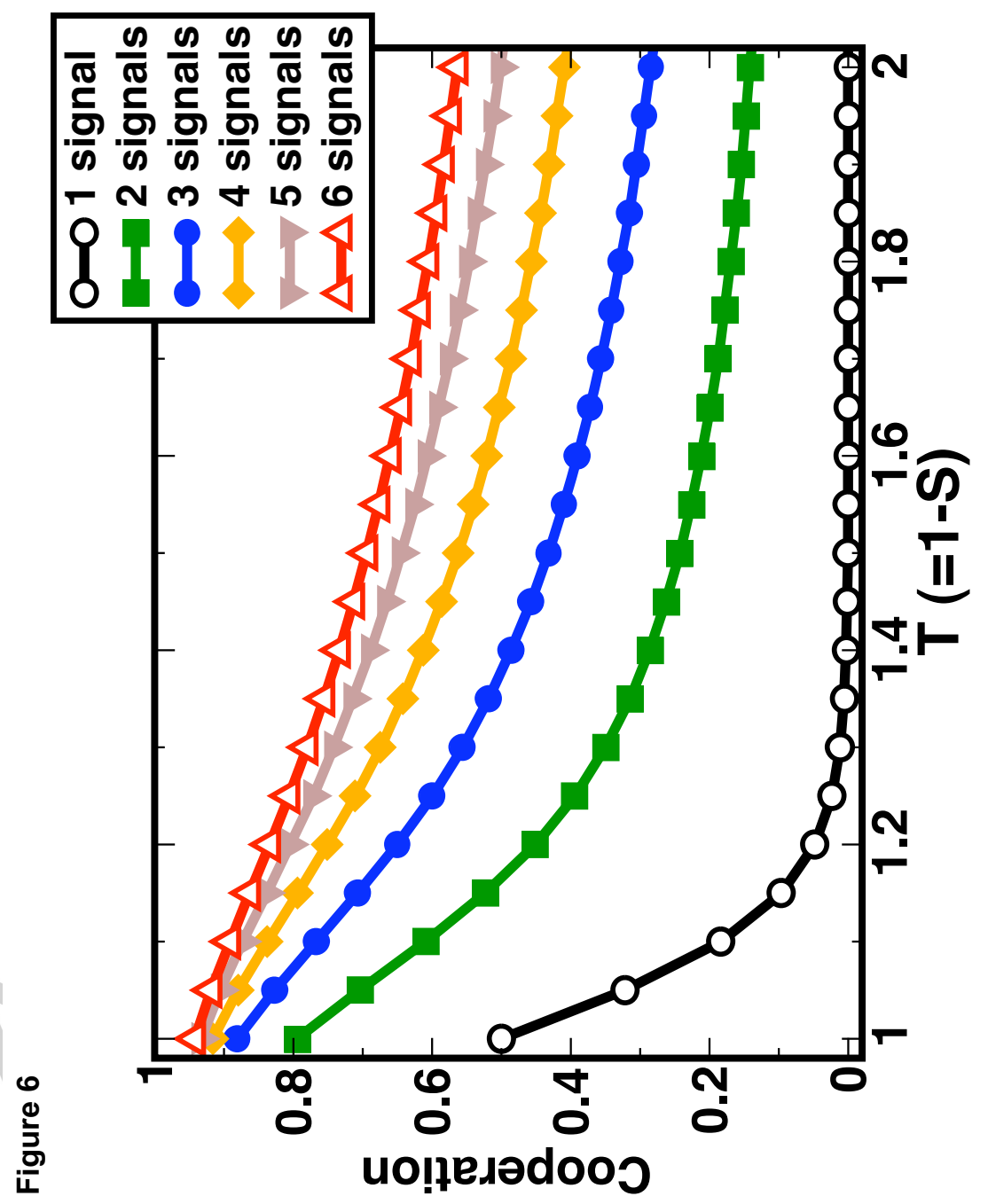

